\title{
COUPLING IMPEDANCES FOR CORRUGATED BEAM PIPES FROM IMPEDANCE BOUNDARY CONDITIONS
}

\author{
S. Petracca, Th. Demma, University of Sannio at Benevento, Italy and INFN
}

\begin{abstract}
An equivalent wall impedance describing the electromagnetic boundary conditions at corrugated pipe walls is introduced in the context of a general perturbative approach for computing the longitudinal and transverse beam coupling impedances in complex heterogeneous pipes.
\end{abstract}

\section{INTRODUCTION}

Coupling impedances are a powerful tool for studying the interaction between a charged particle beam and the surrounding chamber. Unfortunately, coupling impedances can be usually computed only by numerical methods leading to computationally intensive design optimization procedures.

The combined occurrence of complex geometrical features and/or the use of several different wall materials, make the electromagnetic boundary value problem analytically almost untractable. As a matter of fact, only a few analytic solutions for coupling impedances are available, for simple cases where, e.g., the Laplacian is separable in the pipe cross-section coordinates, and the boundary conditions are very simple too (e.g., perfect conductors).

In this paper we estimate the longitudinal and transverse coupling impedances for a pipe with corrugated walls using the general framework presented in [1] and summarized below, using an impedance boundary condition (b.c.) of the Leontóvich type, to account for the corrugations. An application to a candidate LHC geometry is included.

\section{COUPLING IMPEDANCES IN COMPLEX PIPES}

According to [1] the longitudinal and transverse beam coupling impedances $Z_{0, \|}(\omega)$ and $\bar{Z}_{0, \perp}(\omega)$ of a simple, unperturbed pipe (e.g., circular, perfectly conducting) assumed known, can be related to those $Z_{\|}(\omega), \bar{Z}_{\perp}(\omega)$ of another pipe differing from the former by some perturbation in the boundary geometry and/or constitutive properties, as follows (beam at $\vec{r}=0)^{1}$ :

$$
\begin{array}{r}
Z_{\|}(\omega)-Z_{0, \|}(\omega) \mid=\frac{\epsilon_{0}}{\beta_{0} c Q^{2}}\left\{Y_{0} \oint_{\partial S} Z_{w a l l} E_{0 n}^{(i r r .) *}(\vec{r}) .\right. \\
\left.\left[\beta_{0} E_{n}^{(i r r .)}(\vec{r})+\beta_{0}^{-1} E_{n}^{(s o l .)}(\vec{r})\right] d \ell-\oint_{\partial S} E_{0 z}^{*}(\vec{r}) E_{n}^{(i r r .)}(\vec{r}) d \ell\right\},
\end{array}
$$

\footnotetext{
${ }^{1}$ The beam impedances are obviously independent of the total beam charge, as the field in (1) is proportional to $Q$.
}

$$
\begin{gathered}
\overline{\bar{Z}}_{\perp}(\omega)-\overline{\bar{Z}}_{0, \perp}(\omega)=\frac{\epsilon_{0}}{\beta_{0} c Q^{2} k}\left\{Y_{0} \oint_{\partial S} Z_{\text {wall }} \nabla_{\vec{r}_{0}} E_{0 n}^{(i r r .) *}\left(\vec{r}, \vec{r}_{0}\right) \otimes\right. \\
\otimes \nabla_{\vec{r}_{1}}\left[\beta_{0} E_{n}^{(i r r .)}\left(\vec{r}, \vec{r}_{1}\right)+\beta_{0}^{-1} E_{n}^{(s o l .)}\left(\vec{r}, \vec{r}_{1}\right)\right] d \ell+ \\
\left.-\oint_{\partial S} \nabla_{\vec{r}_{0}} E_{0 z}^{*}\left(\vec{r}, \vec{r}_{0}\right) \otimes \nabla_{\vec{r}_{1}} E_{n}^{(i r r .)}\left(\vec{r}, \vec{r}_{1}\right) d \ell\right\}_{\vec{r}_{1}=\vec{r}_{0}=0},
\end{gathered}
$$

where $c=\left(\epsilon_{0} \mu_{0}\right)^{-1 / 2}$ is the speed of light in vacuum, $Y_{0}=\left(\epsilon_{0} / \mu_{0}\right)^{1 / 2}$ is the vacuum characteristic admittance, $\epsilon_{0}$ and $\mu_{0}$ being the vacuum permittivity and permeability, $\beta_{0}$ is the relativistic factor, $Q$ is the total beam charge, $\vec{E}^{(s o l .)}, \vec{E}^{(i r r .)}$ are the solenoidal and irrotational parts of the electric field, a suffix " 0 " identifies the unperturbed quantities, and an impedance (Leontóvich) boundary condition is assumed to hold at the (perturbed) pipe wall $\partial S$ :

$$
\hat{u}_{n} \times\left(\hat{u}_{n} \times \vec{E}-Z_{\text {wall }} \vec{H}\right)_{\partial S}=0,
$$

where $Z_{\text {wall }}$ is the pipe-wall complex characteristic impedance and $\hat{u}_{n}$ is the unit vector normal to $\partial S$.

The first integral term on the r.h.s of (1) and (2) is nonzero if and only if $Z_{\text {wall }}$ is not identically zero on $\partial S$, and accounts for the effect of the (complex) wall conductivity. The second integral term on the r.h.s. of (1) and (2), on the other hand, accounts for the effect of the geometrical perturbation of the boundary, and is non-zero if and only if the unperturbed axial field component $E_{0 z}$ is not identically zero on $\partial S$. Letting $\vec{E}_{0}$ in place of $\vec{E}$ in (1) and (2), one obtains a first order perturbative formula for the beam coupling impedances in the perturbed pipe.

\section{CORRUGATED BEAM PIPES}

Let

$$
\vec{r}=\vec{r}_{b}(\theta)=\vec{R}_{b}(\theta)+\delta \vec{R}(\theta),
$$

the (transverse) position of a point on the (perturbed) pipe boundary $\partial S$, where $\vec{R}_{b}(\theta)$ defines the unperturbed boundary $\partial S_{0}, \delta \vec{R}(\theta)$ describes the $z$-independent roughness, and $\theta$ is the polar angle. To first order in the corrugations,

$$
E_{0 z}^{*}\left(\vec{r}_{b}\right) \sim E_{0 z}^{*}\left(\vec{R}_{b}\right)+\left.\nabla E_{0 z}^{*}\right|_{\vec{R}_{b}} \cdot \delta \vec{R} .
$$

The first term in (5) is obviously zero (the unperturbed boundary is by assumption a perfect conductor). The unperturbed longitudinal field is related to the potential $\Phi_{0}$,

$$
E_{0 z}^{*}=-j k\left(1-\beta_{0}^{2}\right) \Phi_{0}^{*},
$$


whereby

$$
\vec{E}_{0}^{*}=-\nabla \Phi_{0}^{*}
$$

so that (5) becomes

$$
E_{0 z}^{*}\left(\vec{r}_{b}\right) \sim j k\left(1-\beta_{0}^{2}\right) E_{0 n}^{*}\left(\vec{R}_{b}\right) \hat{u}_{n 0}(\theta) \cdot \delta \vec{R} .
$$

since the tangential component of $\vec{E}_{0}$ at the unperturbed boundary (perfect conductor) is zero .

Accordingly, the integral in (1) which accounts for the effects of the geometrical perturbation of the pipe boundary can be written, to first order:

$$
I_{\|}=-j k\left(1-\beta_{0}^{2}\right) \oint_{\delta S_{0}} \hat{u}_{n}(\ell) \cdot \delta \vec{R}(\ell)\left|E_{0 n}^{*}(\ell)\right|^{2} d \ell
$$

where $\ell$ is a curvilinear coordinate on $\delta S_{0}$.

Similarly, to first order in the corrugation term $\delta \vec{R}$,

$$
\begin{gathered}
\nabla_{\vec{r}_{0}} E_{0 z}^{*}\left(\vec{r}_{b}, \vec{r}_{0}\right) \otimes \nabla_{\vec{r}_{1}} E_{0 n}\left(\vec{r}_{b}, \vec{r}_{1}\right) \\
\approx j k\left(1-\beta_{0}^{2}\right) \delta \vec{R} \cdot \hat{u}_{n 0}(\theta) \\
\cdot\left[\nabla_{\vec{r}_{0}} E_{0 n}^{*}\left(\vec{R}_{b}, \vec{r}_{0}\right) \otimes \nabla_{\vec{r}_{1}} E_{0 n}\left(\vec{R}_{b}, \vec{r}_{1}\right)\right] .
\end{gathered}
$$

Accordingly, the integral in (2) which accounts for the effects of the geometrical perturbation of the pipe boundary can be written, to first order:

$$
\begin{gathered}
I_{\perp}=-j k\left(1-\beta_{0}^{2}\right) \oint_{\partial S_{0}} \hat{u}_{n}(\ell) \cdot \delta \vec{R}(\ell) \\
\cdot\left\{\nabla_{\vec{r}_{0}} E_{0 n}^{*}\left(\ell, \vec{r}_{0}\right) \otimes \nabla_{\vec{r}_{1}} E_{0 n}\left(\ell, \vec{r}_{1}\right)\right\}_{\vec{r}_{1}=\vec{r}_{0}=0} d \ell
\end{gathered}
$$

Comparison of (9), (11) to (1) and (2) shows that the roughness $\delta \vec{R}(\theta)$ is "equivalent" to a non-uniform, purely reactive impedance loading

$$
Z_{\text {wall }}^{(\text {equiv. })}=-j k\left(1-j \beta_{0}^{2}\right) Z_{0} \hat{u}_{n 0}(\theta) \cdot \delta \vec{R}(\theta),
$$

laid down on the unperturbeded pipe wall. It is also seen that, for the special case where $\delta R(\theta)$, is a random process, its statistical moments are simply related to those of the equivalent wall-impedance (12). These findings are more or less obviously related to the general formalism developed in [3] for describing (weakly) irregular surfaces in terms of impedance boundary conditions.

\section{CORRUGATED CIRCULAR PIPE}

As a simplest example, we refer to a corrugated perfectly conducting circular pipe. The unperturbed geometry is a smooth perfectly conducting pipe of radius $R$. The unperturbed field produced at $\vec{r}$ by a beam at $\vec{r}_{0}$ is ${ }^{2}$

$$
\vec{E}_{0}\left(\vec{r}, \vec{r}_{0}\right)=\frac{Q}{2 \pi \epsilon_{0}}\left\{\frac{\vec{r}-\vec{r}_{0}}{\left|\vec{r}-\vec{r}_{0}\right|^{2}}-\frac{\vec{r}-\vec{r}_{0}\left(R / r_{0}\right)^{2}}{\left|\vec{r}-\vec{r}_{0}\left(R / r_{0}\right)^{2}\right|^{2}}\right\},
$$

\footnotetext{
${ }^{2}$ The field (13) is the vacuum field produced by beam at $\vec{r}_{0}$, and its image at $\vec{r}_{0}\left(R / r_{0}\right)^{2}$.
}

From (13) one readily obtains

and:

$$
\vec{E}_{0}(\vec{r})=\frac{Q}{2 \pi \epsilon_{0}} \frac{\vec{r}}{|\vec{r}|^{2}}
$$

$$
\lim _{r_{0} \rightarrow 0} \nabla_{\vec{r}_{0}} \vec{E}_{0}\left(\vec{r}, \vec{r}_{0}\right)=\lim _{r_{1} \rightarrow 0} \nabla_{\vec{r}_{1}} \vec{E}_{0}\left(\vec{r}, \vec{r}_{1}\right)=\frac{Q}{\pi \epsilon_{0}} \frac{\vec{r}}{r^{3}} .
$$

Now consider the perturbed case of a circular pipe with uniform wall impedance $Z_{\text {wall }}$. Using eq.s (1) and (2) with $\vec{E}=\vec{E}_{0}$ together with (14) and (15), one readily obtains

$$
Z_{\|}=\frac{Z_{\text {wall }}}{2 \pi R}, \overline{\bar{Z}}_{\perp}=\frac{Z_{\text {wall }}}{\pi k_{0} R^{3}}\left(\hat{u}_{x} \hat{u}_{x}+\hat{u}_{y} \hat{u}_{y}\right),
$$

in agreement with the known exact result [2]. One is therefore led to guess that eq.s (9), (11) should be likewise accurate for computing the couipling impedances contributed by corrugations. Hence, for a perfectly conducting pipe

$$
Z_{\|}=\frac{\left\langle Z_{\text {wall }}^{(\text {equiv. })}\right\rangle}{2 \pi R}, \overline{\bar{Z}}_{\perp}=\frac{\left\langle Z_{\text {wall }}^{(\text {equiv. })}\right\rangle}{\pi k_{0} R^{3}}\left(\hat{u}_{x} \hat{u}_{x}+\hat{u}_{y} \hat{u}_{y}\right),
$$

where

$$
\left\langle Z_{\text {wall }}^{(\text {equiv. })}\right\rangle=-j k\left(1-\beta^{2}\right) Z_{0} \frac{\oint_{\delta S_{0}} \hat{r} \cdot \delta \vec{R}(\ell) d \ell}{2 \pi R}
$$

is the circumferential average of (12). It is seen that suitable ( $z$-independent) corrugations can be used to compensate the remaining reactive terms in the beam coupling impedance at a specific frequency.

\section{LHC IMPEDANCE BUDGET}

The candidate LHC geometry includes two corrugated sections as shown in Fig.1 below. The corrugations con-

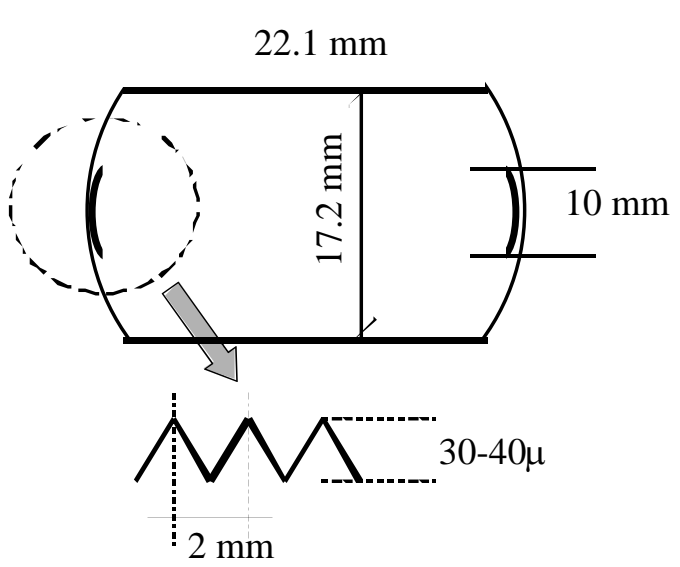

Figure 1: A simplified candidate LHC geometry.

tributions to the (reactive) impedance budget can be easily computed using (13) for the unperturbed field, with $R \approx 14 \mathrm{~mm}$. Assuming $\beta \approx 0.987$, it is found from (18) and Fig.1 that $\left\langle X_{\text {wall }}^{(\text {equiv. })}\right\rangle \approx-1.467 \cdot 10^{-8} \mathrm{ohm}$ at the beam circulation frequency $(\approx 11 \mathrm{KHz})$. 
Proceedings of the 2003 Particle Accelerator Conference

\section{REFERENCES}

[1] S. Petracca, Particle Accel., 50, 211, 1995.

[2] A.W. Chao, Physics of Collective Beam Instabilities in High

Energy Accelerators, J. Wiley \& Sons, 1993.

[3] T.B.A. Senior, Appl. Sci. Res. B8; 437, 1960. 